\title{
De grupos guerrilleros a partidos políticos, un análisis a partir de la cultura política y el desempeño electoral: casos Guatemala y Uruguay.
}

\author{
From Guerrilla Groups to Political Parties. An Analysis based on Political Culture \\ and Electoral Performance: Guatemala and Uruguay Cases
}

Recibido: 11 de diciembre de 2017 - Revisado: 29 de octubre de 2018 - Aceptado: 15 de noviembre de 2018

\section{Brian Nicolás González Moreira ${ }^{1}$ \\ Diego Fernando Torres Araque ${ }^{2}$}

\section{Resumen}

En la actualidad, en Guatemala y Uruguay existen partidos políticos derivados de la desmovilización de grupos guerrilleros. Estos han logrado diferentes niveles de éxito en los procesos electorales como posible consecuencia de los rasgos de cultura política y las características propias de la democracia en los dos casos de estudio. Con el objetivo de aportar en la comprensión de estas diferencias, el presente artículo analizará la relación entre la cultura política y el desempeño electoral de la Unidad Revolucionaria Nacional Guatemalteca (URNG) y el Frente Amplio. Se busca determinar cómo los rasgos de la cultura política han facilitado o dificultado el desempeño electoral de los antiguos grupos guerrilleros y de esta forma dar una aproximación a lo que podrían enfrentar las últimas guerrillas desmovilizadas en Latinoamérica.

\section{Palabras clave}

Democracia, cultura política, partidos políticos, grupos guerrilleros, Guatemala, Uruguay, procesos de paz, reintegración.

\begin{abstract}
IIn Guatemala and Uruguay, there are currently political parties derived from the demobilization of guerrilla groups. These have achieved different levels of success in electoral processes as a possible consequence of their distinctive traits of political culture and their democracies' characteristics. To contribute to the understanding of the differences between the two cases of study, this article analyzed the relationship between the political culture and the electoral performance of the Unidad Revolucionaria Nacional Guatemalteca-URNG and the Frente Amplio. The aim was to determine how the traits of the political culture have facilitated or hindered the electoral performance of the former guerrilla groups and, therefore, to give an approximation to what the last demobilized guerrillas in Latin America might face.
\end{abstract}

\section{Keywords}

Democracy, political culture, political parties, guerrilla groups, Guatemala, Uruguay, peace process, reenter society.

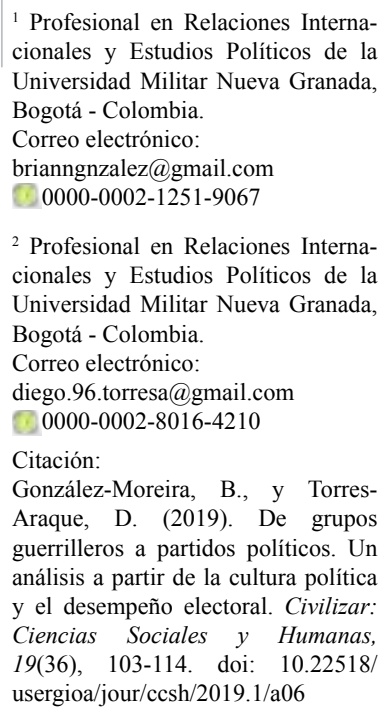

${ }^{1}$ Profesional en Relaciones Internacionales y Estudios Políticos de la Universidad Militar Nueva Granada, Bogotá - Colombia.

Correo electrónico:

brianngnzalez@gmail.com

0000-0002-1251-9067

${ }^{2}$ Profesional en Relaciones Internacionales y Estudios Políticos de la Universidad Militar Nueva Granada, Bogotá - Colombia.

Correo electrónico:

diego.96.torresa@gmail.com

0000-0002-8016-4210

Citación:

González-Moreira, B., y TorresAraque, D. (2019). De grupos guerrilleros a partidos políticos. Un análisis a partir de la cultura política y el desempeño electoral. Civilizar: Ciencias Sociales y Humanas, 19(36), 103-114. doi: 10.22518/ usergioa/jour/ccsh/2019.1/a06 


\section{Introducción}

La cultura política logra definir aspectos fundamentales de la democracia de un país.

Guatemala vivió desde la década de los 40 una sucesión de dictaduras militares que reprimieron las libertades individuales y persiguieron, a través de la violencia, cualquier forma de oposición política. Por otra parte, en el marco de la guerra fría y por la influencia norteamericana, se utilizaron estrategias contrainsurgentes que incluyeron la conformación de grupos paramilitares (Patrullas de Autodefensa Civil). Ello desembocó en un estado de lucha constante contra cualquier manifestación o movimiento que pudiera ser catalogado como comunista o de izquierda (Giraldo, 2004).

Las primeras guerrillas guatemaltecas fueron el Ejército Guerrillero de los Pobres, la Organización del Pueblo en Armas, el Partido Guatemalteco del Trabajo y las Fuerzas Armadas Rebeldes, que luchaban por la defensa del sector rural e indígena. Estas guerrillas se unificaron en 1980 y conformaron la Unidad Revolucionaria Nacional Guatemalteca. Entre sus reivindicaciones se encuentra la protección de los derechos de la población campesina e indígena, el respeto por el manejo agrario autónomo y la lucha contra un régimen político excluyente y represivo (Molden, 2015).

La creación de la URNG así como de las patrullas a principios de la década de los 80 , llevó al recrudecimiento del conflicto armado. En este contexto, se dan los primeros acercamientos que llevan al inicio de los diálogos. Entre los factores que facilitaron este proceso es importante mencionar lo siguiente: primero, la conformación del grupo Contadora ${ }^{1}$ que tenía como objetivo poner fin a los conflictos derivados de procesos foráneos que se extendían en Centroamérica y también promover la democratización y la cooperación en la región. Segundo, la conformación de un proceso de diálogo nacional auspiciado por la Comisión
Nacional de Reconciliación. Tercero, la entrada en vigencia de una nueva Constitución en 1985 que amplía los espacios de participación y establece las garantías básicas de una democracia liberal. Lo anterior sumado a la celebración de elecciones democráticas, que llevaron a la presidencia a Marco Vinicio Cerezo, civil, promotor y firmante de los acuerdos de Esquipulas (Alba, Suárez, Rueda, Gago, Jaramillo, Moreno y Correal, 2016).

Tras un proceso de negociación largo y lleno de barreras políticas, judiciales y sociales, en 1996 se logra la firma del Acuerdo de Paz Firme y Duradera, donde la URNG se compromete a actuar en legalidad. En 1998 se convierten en partido político y en 1999 participa por primera vez en las elecciones (Giraldo, 2004).

A mediados de los años 60, Uruguay estuvo inmerso en una fuerte crisis económica que llevó al surgimiento de movilizaciones estudiantiles y obreras. De forma simultánea, se vivió la gran expansión de movimientos guerrilleros alrededor de América Latina, teniendo como foco de inspiración la revolución cubana que, gracias a su éxito, tuvo replicabilidad en la región y brindó una perspectiva diferente, teórica y práctica, de lo que debía ser la nueva izquierda latinoamericana; estos movimientos adoptarán la violencia como característica fundamental (Álvarez y Rey, 2012). En este contexto nace el Movimiento de Liberación Nacional - Tupamaros (MLN-T), como respuesta a los acontecimientos coyunturales de la época. Este grupo pretendía emular las consignas de la revolución cubana al llevar a cabo acciones más allá de la teoría. Así, se fundamentó en una ideología heterogénea y, en consecuencia, se reunieron revolucionarios de diferentes tradiciones políticas y teóricas, entre ellos marxismo, leninismo, anarquismo, liberalismo y nacionalismo (Garcé, 2011). Teniendo en cuenta lo anterior, los Tupamaros siempre se caracterizaron por ser una organización flexible y no un movimiento rígido con jerarquías incuestionables y una doctrina política definitiva (Rey, 2006; Garcé, 2006). 
El MLN-T fue desmantelado en 1972 como antesala a la dictadura de Juan María Bordaberry. Para 1985, cuando se restablece la democracia, los últimos presos políticos fueron liberados, entre ellos Pepe Mujica y Raúl Sendic, líderes tradicionales de la organización, quienes habían sido encarcelados en condiciones especialmente brutales durante más de una década (Martí, Garcé y Martín, 2013). Al ser liberados, toman la decisión de actuar en la legalidad y de aprovechar los nuevos espacios de apertura política que se presentaban por el fin de la dictadura (Garcé, 2006).

Después de varias discusiones entre miembros del movimiento, motivadas por las diferentes perspectivas respecto a la reestructuración ideológica del grupo, a comienzos de 1989 los Tupamaros toman la decisión de ingresar formalmente a la coalición de izquierda Frente Amplio y formar el Movimiento de Participación Popular (MPP) (Martí, Garcé y Martín, 2013).

El Frente Amplio surge en el año 1971 como una coalición de partidos, en su mayoría de izquierda, que buscaba hacer frente al bipartidismo liderado por el Partido Colorado y el Partido Nacional. Los partidos que hicieron parte de esta coalición fueron: Partido Socialista, Partido Comunista, grupos independientes, Democracia Cristiana y colectivos de personas que se apartaron de los partidos tradicionales. Como se explicó anteriormente, en 1989 el MPP entró a ser parte de la coalición del Frente Amplio como una de sus fracciones. En la actualidad, el Frente Amplio es un partido de coalición en donde sus fracciones ya no son partidos autónomos, sino partes que componen una sola unidad (Lanzaro, 2004; Martí, Garcé y Martín, 2013).

En un principio esta nueva alternativa política no recibió un amplio apoyo por parte de la ciudadanía, sin embargo, con el tiempo incrementó su respaldo político y el MPP se convirtió en la facción más importante del partido
Frente Amplio. Algunos de los pilares ideológicos característicos del MLN-T fueron fundamentales para adaptación de esta organización como partido. Entre ellos el pragmatismo que legitimó los sucesivos cambios de rumbo, el pluralismo que permitió la adhesión de diferentes sectores, que los líderes contaran con espacios de autonomía para desplegar sus propias políticas y el nacionalismo que facilitó la conexión del discurso con las principales tradiciones culturales y populares del país (Martí, Garcé y Martín, 2013).

Este artículo busca analizar la relación entre la cultura política y el proceso de reincorporación a la vida política legal de la Unidad Revolucionaria Nacional Guatemalteca (URNG) y el Movimiento de Liberación Nacional - Tupamaros (MLN-T) en Uruguay. Más específicamente, se pretende determinar en qué medida los rasgos de la cultura política, entendida como el resultado de las relaciones en los niveles macro y micro de la política para Almond y Verba (1970), han incidido en el desempeño electoral de la URNG y el MLN-T una vez que se convirtieron en partidos políticos legales que interactúan con la sociedad guatemalteca y uruguaya. Determinar la influencia de la cultura política en el desempeño electoral de un grupo guerrillero que se reintegra a la vida civil, como actor político legal, y entra a competir por cargos públicos mediante elección popular, es el problema que subyace al estudio planteado en este artículo. Esto con el fin de exponer dos escenarios diferentes que pueden llegar a ser recreados en procesos de reintegración y participación política legal de las últimas guerrillas latinoamericanas.

Teniendo en cuenta lo anterior y con el propósito de comparar el proceso de adaptación partidistas de los casos mencionados, este documento se divide en cuatro secciones. La primera plantea una discusión teórica sobre la cultura política y su relación con la democracia. En la segunda sección se compara el desempeño electoral a escala nacional de los movimientos 
políticos analizados. En la tercera parte se discuten los principales rasgos de la cultura política de Guatemala y Uruguay a partir de unas variables específicas que se definen en el método. En la cuarta y última sección se presentan las conclusiones del artículo, donde se plantea la existencia de una relación directa entre los niveles de democracia, los rasgos de cultura política y el desempeño electoral de la URNG y el Frente Amplio.

\section{Método}

Se estudian los casos de Guatemala y Uruguay, porque constituyen ejemplos claros de éxito y fracaso en el proceso de reincorporación a la legalidad de grupos guerrilleros, al considerar el desempeño electoral y la calidad de su democracia.

Para comprender el proceso de adaptación partidista de la URNG y los Tupamaros, se utilizaron dos fuentes de información principales. En primer lugar, los resultados de las elecciones nacionales en Guatemala y Uruguay, a partir del momento en que los grupos estudiados se incorporan a la vida política legal (ver Tabla 1). En segundo lugar, las encuestas sobre cultura política, adelantadas por el Latinobarómetro (2016) y el Proyecto de Opinión Pública de América Latina -LAPOP- (2016).

Respecto al Latinobarómetro, la Tabla 2 presenta las variables escogidas para comparar los principales rasgos de la cultura política en los países estudiados.

Para el caso de LAPOP se escogieron dos variables de comparación. En primer lugar, el apoyo al sistema definido como el grado de legitimidad que le reconoce la sociedad a las instituciones. En segundo lugar, la tolerancia política entendida como la aceptación de principios de competencia democrática, además de la tolerancia y el respeto frente a los derechos de los disidentes. La relación entre estas variables es importante para el análisis, pues indica en qué medida los ciudadanos tienen actitudes conducentes a una democracia estable (LAPOP, 2016).

LAPOP genera una escala de 100 puntos para las dos variables. Si el país obtiene más de 50 puntos tanto en apoyo al sistema como en tolerancia politica, se considera una democracia estable. Por el contrario, si obtiene una calificación por debajo de 50 puntos en las dos variables, se clasifica como una democracia en riesgo. En los casos donde se observe más de 50 puntos en tolerancia política y menos de 50 en apoyo al sistema se cataloga como una democracia inestable. Por último, si el país tiene más de 50 puntos en apoyo al sistema y menos de 50 en tolerancia política se define como una estabilidad autoritaria (LAPOP, 2016).

\section{Cultura política y democracia}

Se entiende como cultura política al conjunto de valores, actitudes, orientaciones, percepciones o creencias de los ciudadanos frente al sistema político (Sabetti, 2009). Orientaciones que según Almond y Verba (1970) pueden ser de tres tipos: orientaciones cognitivas, que se refieren a los conocimientos y creencias acerca del sistema político, los roles que este desempeña y los inputs y outputs que resultan del proceso político; orientaciones afectivas, que

Tabla 1

Fuentes de consulta de resultados electorales

\begin{tabular}{c|c|c|c} 
País & Fuente & Periodo & Página web \\
\hline Guatemala & Tribunal Supremo Electoral & $1996-2016$ & http://tse.org.gt/ \\
\hline Uruguay & Corte Electoral & $1990-2015$ & http://www.corteelectoral.gub.uy/gxpsites/page.aspx
\end{tabular}

Fuente: Elaboración propia. 
Tabla 2

Variables escogidas del informe Latinobarómetro para el año 2014

\begin{tabular}{|c|c|}
\hline Variable & Descripción \\
\hline Apoyo a la democracia & $\begin{array}{l}\text { Mide la preferencia de la democracia sobre cualquier otra forma de gobierno. Es una } \\
\text { variable general y explícita, que además se ve afectada por las crisis económicas. }\end{array}$ \\
\hline Democracia Churchilliana & $\begin{array}{c}\text { Esta variable se basa en una frase del ex Primer Ministro británico Winston Churchill: "La } \\
\text { democracia puede tener problemas, pero es el mejor sistema de gobierno". Se trata de una } \\
\text { variable de carácter más difuso. }\end{array}$ \\
\hline Satisfacción con la democracia & $\begin{array}{l}\text { Mide la percepción de la sociedad frente al funcionamiento de los gobiernos y el } \\
\text { desempeño económico. }\end{array}$ \\
\hline Autoritarismo político & $\begin{array}{l}\text { Mide el grado de aceptación de un gobierno autoritario, preguntando a la ciudadanía si se } \\
\text { encuentra o no de acuerdo con la siguiente frase: "No me importa un gobierno democrático } \\
\text { que llegue al poder si puede resolver problemas". }\end{array}$ \\
\hline Autoritarismo social & $\begin{array}{l}\text { Refleja la cultura, actitudes y comportamientos de las personas en la vida cotidiana. Esta } \\
\text { variable mide la demanda de "mano dura" de los ciudadanos hacia el gobierno. }\end{array}$ \\
\hline Confianza interpersonal & Mide el grado de confianza que tienen los ciudadanos hacia los demás.- \\
\hline Orden social vs. Libertad individual & $\begin{array}{c}\text { Este indicador mide en qué medida los ciudadanos prefieren una sociedad ordenada } \\
\text { donde se controlen problemas como la delincuencia y la violencia, incluso si eso significa } \\
\text { sacrificar las libertades individuales. }\end{array}$ \\
\hline Confianza en partidos políticos & Mide el grado de confianza de la población frente a los partidos políticos. \\
\hline
\end{tabular}

Fuente: Elaboración propia según información del Latinobarómetro.

involucran sentimientos acerca del sistema político y sus diferentes componentes y orientaciones evaluativas, que tienen en cuenta los juicios y opiniones de los ciudadanos sobre los objetos políticos, y que usualmente combinan criterios de valor con información y sentimientos.

La cultura política influye en el nivel de legitimidad del régimen político, pues el tipo de orientaciones predominantes en la ciudadanía (en especial las afectivas/valorativas y las evaluativas) afecta la evaluación sobre el desempeño del régimen (incluidos los actores y las instituciones); lo que a su vez se refleja de manera directa en el apoyo de la ciudadanía frente al sistema político. Por lo tanto, es importante tener en cuenta la legitimidad en los estudios sobre la cultura política y medirla a través de encuestas de opinión pública que registren las percepciones de los ciudadanos (Santana, 1998).

La cultura política tiene una incidencia importante en el funcionamiento de los sistemas democráticos, pues el sostenimiento de la democracia no solo se supedita a la existencia de instituciones como los partidos políticos, el sufragio universal y los congresos, sino que también depende de la existencia de una cultura política donde los principios y creencias propias de la democracia se encuentran arraigados entre la ciudadanía y sean aceptados (Almond y Verba, 2001).

En un repaso histórico sobre el concepto de la cultura política, Rodríguez (2017), reconoce un antecedente valioso en la obra de Alexis de Tocqueville sobre la democracia en los Estados Unidos y Francia. Allí se resalta la importancia de las costumbres y de las subculturas políticas que explican la estabilidad democrática norteamericana, así como la acción política de campesinos, burgueses y aristócratas durante la Revolución Francesa; postulado que fortalece la relación entre cultura política y estabilidad democrática.

En este mismo sentido, Folcher (2000) considera que la cultura política es un factor determinante para alcanzar la estabilidad y fortaleza de las democracias modernas. Estas no dependen solo de la justicia que se imparte entre los miembros de la sociedad, sino que las 
cualidades y actitudes de los ciudadanos sientan las bases para el óptimo desarrollo del sistema político democrático.

Por su parte, Leticia Heras (2004) también reconoce la importancia de la cultura política en los procesos de consolidación democrática en América Latina. Para esta autora, la cultura política es una noción histórica social que varía según el contexto de cada país y la forma en que se consolidan los valores democráticos en su interior. Razón por la cual la cultura política no es estática, sino que sufre transformaciones lentas relacionadas con determinados cambios sociales.

Dada la relación entre democracia y cultura política que se expuso en los párrafos anteriores, esta última constituye un elemento importante para comprender el éxito o fracaso en la implementación de los acuerdos que se derivan de los procesos de paz, en especial en lo relativo a la participación política de los grupos alzados en armas que se desmovilizan y se integran al proceso político legal.

Desde una perspectiva psicológica hay una relación entre la cultura política y los imaginarios y mentalidades de la sociedad. Las realidades que construyen los diferentes grupos sociales llevan a la aceptación o el rechazo de sectores de izquierda, derecha, oficialismo u oposición, según el caso, y por tanto influyen en el apoyo a un sistema democrático o autoritario (López, 2000).

En tal sentido, la inserción exitosa a la vida política legal de antiguos grupos guerrilleros dependerá también de la cultura política dentro de los respectivos sistemas políticos. Así, una cultura política caracterizada por rasgos como la tolerancia, la solidaridad, el respeto por las opiniones diferentes y la confianza en las instituciones democráticas podrían facilitar la participación electoral de los grupos guerri1leros desmovilizados. Por el contrario, la ausencia de este tipo de rasgos podría dificultar el desempeño electoral de los mencionados grupos. En otras palabras, las actitudes y creencias de los ciudadanos explican el desempeño electoral de los diferentes grupos guerrilleros que se han incorporado a la vida política legal.

\section{Desempeño electoral de la URNG y el Frente Amplio}

La primera variable que se evalúa hace referencia a los resultados electorales a nivel nacional que obtuvieron la URNG y el Frente Amplio.

En lo que respecta al poder ejecutivo (ver Tabla 3), la URNG ha recibido un escaso apoyo electoral. En 1999 participaron por primera vez en las elecciones presidenciales con el candidato Álvaro Colom y obtuvieron el 12,3\% de los votos. Desde entonces, la tendencia que se observa es la reducción constante en el caudal electoral, pues su porcentaje de votación no superó el 3\% de los votos en las últimas cuatro elecciones presidenciales. Para 2015 tan solo lograron un 2,6\% de la votación nacional, cuando presentaron como candidato a Miguel Ángel Sandoval en la coalición WINAQ-URNGMAIZ (Asociación de Investigación y Estudios Sociales - ASIES, 2016).

A diferencia de la URNG, el Frente Amplio contó desde el principio con una base electoral que le permitió obtener el 20,3\% de la votación en las elecciones presidenciales de 1990, en las que participó con candidatos exmiembros del antiguo MLN-T. En 2005, el partido logró ampliar su margen de aceptación y ganó por primera vez la presidencia de la República con el candidato Tabaré Vázquez. Posteriormente, en el año 2010 ganaron de nuevo las elecciones presidenciales con el candidato José Mujica, uno de los principales dirigentes del antiguo MLN-T. En las últimas elecciones celebradas en el país, Tabaré Vázquez se presentó de nuevo y obtuvieron por tercera vez la presidencia, lo que los consolida como la primera fuerza política del país (ver Tabla 3). 
Tabla 3

Resultados electorales presidenciales

\begin{tabular}{c|c|r|r} 
País & \multicolumn{1}{|c|}{ Año } & \multicolumn{1}{c|}{ Votos } & Porcentaje \\
Guatemala - URNG & 1999 & 270.891 & $12,3 \%$ \\
& 2003 & 69.297 & $2,5 \%$ \\
& 2007 & 70.208 & $2,1 \%$ \\
& 2011 & 145.080 & $3,2 \%$ \\
& 2015 & 98.633 & $2,0 \%$ \\
\hline Uruguay - FA & 1990 & 418.403 & $20,3 \%$ \\
& 1995 & 621.226 & $29,1 \%$ \\
& 2000 & 982.049 & $45,8 \%$ \\
& 2005 & 1.124 .761 & $50,4 \%$ \\
& 2010 & 1.197 .638 & $54,6 \%$ \\
& 2015 & 1.226 .105 & $56,6 \%$
\end{tabular}

Fuente: Elaboración propia con datos Tribunal Supremo Electoral (Guatemala) y Corte Electoral (Uruguay).

En el poder legislativo se mantiene la tendencia de las elecciones presidenciales. El análisis de los resultados de las elecciones para el Congreso de la República ${ }^{2}$ en Guatemala y la Asamblea General ${ }^{3}$ en Uruguay, indican un bajo desempeño para la URNG, en contraste con los resultados favorables obtenidos por el Frente Amplio.

La URNG ha mantenido una participación mínima al interior del Congreso, su mayor representación se dio en las elecciones de 1999 cuando alcanzó nueve curules. En los siguientes periodos hubo una reducción significativa, al ocupar tan solo un escaño en las últimas elecciones de 2015. Por el contrario, en las elecciones de 1990 el Frente Amplio obtuvo siete curules en el Senado y veintiuna en la Cámara de Representantes. En los períodos siguientes, el número de curules alcanzadas aumentó de manera progresiva y se mantiene estable en las dos últimas elecciones legislativas (ver Tabla 4). Hoy día cuentan con quince curules en el Senado y cincuenta en la Cámara de Representantes, con lo que controlan las mayorías en las dos cámaras de la Asamblea General. Esto, sumado a la continuidad en el poder ejecutivo, ha garantizado importantes niveles de gobernabilidad y ha facilitado la implementación de sus políticas. Entre ellas los planes de emergencia social y equidad, la creación de un nuevo siste- ma integral de salud, una reforma tributaria de tipo progresivo que busca disminuir los niveles de pobreza y exclusión social (Zapirain, s.f.).

\section{Rasgos de la cultura política en Guatemala y Uruguay}

En relación con la cultura política presente en cada país, primero se analizan los resultados de las encuestas de opinión pública del Latinobarómetro, luego la clasificación propuesta por LAPOP con el fin de determinar el conjunto de valores, actitudes, orientaciones, percepciones o creencias de los ciudadanos frente al sistema político (Sabetti, 2009).

Según el Latinobarómetro (2016), el apoyo a la democracia ha sido tradicionalmente bajo en Guatemala. Para el 2016 solo el 31\% de los encuestados consideraban a la democracia como la mejor forma de gobierno. En cuanto a la democracia Churchilliana, que mide de forma más difusa el apoyo a la democracia, el $63 \%$ de la población estuvo de acuerdo con que la democracia podía tener problemas, pero era la mejor forma de gobierno. El 61,1\% manifiesta no sentirse satisfecho con la democracia, variable que indica, ante todo, desempeño económico. Este porcentaje considera que el gobierno no ha respondido a las demandas de la población y que la corrupción ha empeorado. Para el caso de Uruguay, las anteriores variables registran un $68 \%, 86 \%$ y $27,7 \%$ respectivamente, lo que evidencia un mayor respaldo hacia la democracia entre los ciudadanos uruguayos (ver Tabla 5).

Con respecto al autoritarismo político, en Guatemala el $60 \%$ de la población aprueba un gobierno que resuelva sus problemas sociales sin importar que este sea autoritario. En cambio, en Uruguay este porcentaje es del $40 \%$. Para el caso del autoritarismo social, la tendencia se invierte, pues el porcentaje de ciudadanos que demandan mano dura es mayor en Uruguay (71\%) que en Guatemala (63\%) (Ver Tabla 5). 
Tabla 4.

Resultados electorales Congreso

\begin{tabular}{|c|c|c|c|c|c|}
\hline \multirow[b]{3}{*}{ Guatemala - URNG } & Año & Curules & \multicolumn{3}{|c|}{ Porcentaje } \\
\hline & & \multicolumn{3}{|c|}{ Congreso de la República } & \\
\hline & $\begin{array}{l}1999 \\
2003 \\
2007 \\
2011 \\
2015\end{array}$ & & & $\begin{array}{l}7,9 \% \\
1,2 \% \\
1,2 \% \\
1,2 \% \\
0,6 \%\end{array}$ & \\
\hline & & Senado & $\begin{array}{c}\text { Cámara de } \\
\text { Representantes }\end{array}$ & Senado & $\begin{array}{c}\text { Cámara de } \\
\text { Representantes }\end{array}$ \\
\hline Uruguay - FA & $\begin{array}{l}1990 \\
1995 \\
2000 \\
2005 \\
2010 \\
2015\end{array}$ & $\begin{array}{c}7 \\
9 \\
12 \\
16 \\
16 \\
15\end{array}$ & $\begin{array}{l}21 \\
31 \\
40 \\
52 \\
50 \\
50\end{array}$ & $\begin{array}{l}23 \% \\
30 \% \\
40 \% \\
53 \% \\
53 \% \\
50 \%\end{array}$ & $\begin{array}{l}21 \% \\
31 \% \\
40 \% \\
52 \% \\
50 \% \\
50 \%\end{array}$ \\
\hline
\end{tabular}

Fuente: Elaboración propia según datos Tribunal Supremo Electoral (Guatemala) y Corte Electoral (Uruguay).

Tanto en Guatemala como en Uruguay la población prefiere el orden social sobre las libertades individuales. En Guatemala el 54\% elige orden social y solo el $37 \%$ libertad individual; en Uruguay los porcentajes son muy similares con $58 \%$ y $39 \%$ respectivamente (ver Tabla 5).

En el caso de la confianza interpersonal también se encuentran similitudes y en los casos se observan bajas puntuaciones, pues tan solo el $20 \%$ de los guatemaltecos y el $17 \%$ de los uruguayos afirman confiar en el prójimo (ver Tabla 5).

Tabla 5

\begin{tabular}{c|r|r|}
\hline Variables & Guatemala & Uruguay \\
\hline Apoyo a la democracia & $31 \%$ & $68 \%$ \\
\hline Democracia Churchilliana & $63 \%$ & $86 \%$ \\
\hline Satisfacción con la democracia * & $61,1 \%$ & $27,7 \%$ \\
\hline Autoritarismo político & $60 \%$ & $41 \%$ \\
\hline Autoritarismo social & $63 \%$ & $71 \%$ \\
\hline Confianza interpersonal & $20 \%$ & $17 \%$ \\
\hline Orden social & $54 \%$ & $58 \%$ \\
\hline Libertad individual & $37 \%$ & $39 \%$ \\
\hline Confianza en partidos políticos ${ }^{* *}$ & $44 \%$ & $28 \%$ \\
\hline
\end{tabular}

Nota: * Sumatoria de los no muy satisfechos y nada satisfechos con respecto a la democracia. **Porcentaje de desconfianza hacia los partidos políticos. Elaboración propia según datos del informe Latinobarómetro (2014).
En la variable confianza en los partidos politicos, se observan diferencias significativas entre los dos países. En Guatemala el 44\% de la población desconfía de los partidos, mientras que en Uruguay este porcentaje es solo del $28 \%$ (ver Tabla 5).

Por último, respecto al análisis de la cultura política adelantado por LAPOP, Guatemala obtiene 49 puntos en apoyo al sistema y 27,2 en tolerancia política, razón por la cual es considerada como una democracia en riesgo. En Uruguay se observa una situación muy diferente, pues tiene 58,4 puntos de apoyo al sistema y 62,6 de tolerancia política; en tal sentido, es clasificada como una democracia estable. Lo que indicaría un compromiso mucho más sólido de la ciudadanía con respecto al sistema político (ver Tabla 6).

Tabla 6

Resultados cultura política LAPOP

\begin{tabular}{c|r|r|c} 
País & $\begin{array}{c}\text { Apoyo al } \\
\text { sistema }\end{array}$ & $\begin{array}{c}\text { Tolerancia } \\
\text { política }\end{array}$ & Relación \\
Guatemala & 49 & 27,2 & Democracia en riesgo \\
\hline Uruguay & 58,4 & 62,6 & Democracia estable
\end{tabular}

Fuente: Elaboración propia según datos del informe LAPOP (2016). 


\section{Conclusiones}

En este artículo se buscó mostrar la relación que existe entre la cultura política y el proceso de adaptación partidista de la URNG y el Frente Amplio, al comparar su desempeño electoral con los rasgos característicos de cultura política, descritos con base del análisis de las encuestas de opinión pública. A partir de diferentes planteamientos teóricos, se argumentó que la cultura política de un país influye en el grado de apoyo que la sociedad le otorga al régimen democrático y que, a su vez, este apoyo facilita la participación en política de antiguos grupos guerrilleros.

De esta manera, Guatemala presenta una cultura política que se distancia de los rasgos democráticos de los que goza la cultura política uruguaya, lo que ha generado diferencias en cuanto a la solidez del régimen democrático en el que se desenvuelven la URNG y el Frente Amplio. Así, mientras el proceso de incorporación a la vida política legal de la URNG tiene lugar en un régimen híbrido, el del Frente Amplio se da en una democracia plena, según la clasificación elaborada por The Economist (2016).

En cuanto a los resultados electorales, se mostró que desde su aparición como partido político la URNG no ha logrado una participación electoral exitosa. No solo no ha alcanzado una representación significativa en el Congreso de la República, tampoco ha logrado configurarse como una verdadera alternativa de poder en las elecciones presidenciales. Por el contrario, el Frente Amplio ha ganado las elecciones presidenciales en tres ocasiones consecutivas y ha logrado también las mayorías parlamentarias, lo que representa un proceso de adaptación partidista exitoso.

Así mismo, se argumentó que las diferencias en los resultados electorales estarían relacionadas con la cultura política de los respectivos países. De tal forma, según los datos del Latinobarómetro, en Guatemala se observa un menor apoyo hacia la democracia, no existe una satisfacción generalizada con esta forma de gobierno, se observan altos niveles de autoritarismo político y la mayoría de la población desaprueba el desempeño de los partidos políticos. Por su parte, Uruguay tiene una cultura política más pluralista pues los niveles de apoyo a la democracia son más altos, existe un mayor grado de satisfacción con la misma, se encuentra menos arraigado el autoritarismo político y existe una mayor confianza en los partidos. Tendencia que se confirma también con los datos de LAPOP, según los cuales Guatemala, a diferencia de Uruguay, se caracteriza por tener bajos niveles tolerancia política y de apoyo al sistema.

Por último, aun cuando se observan similitudes en variables como el autoritarismo social, la confianza interpersonal y la importancia que se le da al orden social vs. las libertades individuales, la cultura política uruguaya mantiene claros atributos democráticos que no se observan para el caso de Guatemala.

En conclusión, es posible identificar una relación directa entre los niveles de democracia, los rasgos de la cultura política y el desempeño electoral de la URNG y el Frente Amplio, partidos que surgen del proceso de incorporación a la legalidad de antiguos grupos guerrilleros.

\section{Referencias}

Alba, A., Suárez, B., Rueda, B., Gago, E., Jaramillo, F., Moreno, J., y Correal, O. (2016). Experiencias internacionales de paz: Lecciones aprendidas para Colombia. Bogotá, Colombia: Fundación Universidad de Bogotá Jorge Tadeo Lozano.

Almond, G., Cover, F., y Verba, S. (1970). La cultura cívica: estudio sobre la participación política democrática en cinco naciones. [Traducido al español de The Civic Culture: Political Attitudes 
and Democracy in Five Nations]. Madrid, España: Euramérica Fundación FOESSA.

Almond, G., y Verba, S. (2001). La cultura política. En A. Batlle (Ed.). Diez textos básicos de Ciencia Política. Barcelona, España: Ariel.

Asociación de investigación y estudios sociales. (2016). Informe analítico del proceso electoral 2015. Departamento de Investigaciones Sociopolíticas. Guatemala: ASIES 2016.

Constitución Política de la República de Guatemala [Const.]. (1985). Artículo 157. [Título IV]. Congreso de la República de Guatemala. Recuperado de http://www.congreso.gob.gt/marco-legal.php

Constitución Política de la República de Uruguay [Const.]. (1967). Artículos 84, 88 y 94 [Sección V]. Parlamento del Uruguay. Recuperado de https:// parlamento.gub.uy/documentosyleyes/constitucion

Durán, E. (1984). La solución de Contadora para el logro de la Paz en Centroamérica. Estudios Internacionales, 17(68), 537-547. doi: 10.5354/0719-3769.2011.15844.

Folcher, F. (2000). Ciudadanía y multiculturalidad. Algunas reflexiones sobre la obra de Kymlicka. Ponencia VI al Congreso de Antropología Social, Mar del Plata. Argentina: GAP/UMNDP.

Freedom House. (16 de octubre de 2017). Freedom in the world scores. Guatemala profile. Recuperado de https://freedomhouse. org/report/freedom-world/2017/guatemala

Garcé, A. (2006). Donde hubo fuego. El proceso de adaptación del MLN-Tupamaros a la legalidad y a la competencia electoral (1985-2004) [Anexo documental]. Montevideo: Fin de Siglo.

Garcé, A. (2011). Ideologías políticas y adaptación partidaria: El caso del MLN-
Tupamaros (1985-2009). Revista de ciencia política, 31(1), 117-137. doi: 10.4067/S0718-090X2011000100006

Giraldo, J. (2004). Búsqueda de Verdad y Justicia. Seis experiencias en posconflicto. Bogotá, Colombia: CINEP.

Heras, G. L. (2004). Cultura política y democratización en América Latina. México: Universidad Autónoma del Estado de México.

Lanzaro, J. (2004). La izquierda se acerca a los uruguayos y los uruguayos se acercan a la izquierda. Claves de desarrollo del Frente Amplio. Montevideo, Uruguay. Recuperado de: https://journals.openedition.org/cal/7625\#quotation

Latinobarómetro. (2016). Informe Latinobarómetro 2016. Recuperado de http://www. latinobarometro.org/latNewsShow.jsp

López de la Roche, F. (2000). Aproximaciones al concepto de cultura política. Convergencias: Revista de Ciencias Sociales, 7(22), 93-123.

Martí i Puig, S., Garcé, A., y Martín, A. (2013). ¿Liderazgo, organización o ideología? Las diferentes vías de adaptación partidaria de los movimientos guerrilleros. Los casos de Nicaragua, El Salvador y Uruguay. Revista Española de Ciencia Politica, 13(33), 57-79.

Martín, A., y Rey, E. (2012). La oleada revolucionaria latinoamericana contemporánea, 1959-1996. Definición, caracterización y algunas claves para su análisis. Naveg@ mérica. Revista electrónica de la Asociación Española de Americanistas, (9), 1-36.

Molden, B. (2015). La Guerra Civil guatemalteca: Historias y memorias cruzadas en el entorno global de la guerra fría. Anuario de Estudios Centroamericanos, 41, 6791. doi: 10.15517/aeca.v41i1.21840 
Proyecto de Opinión Pública de América Latina [LAPOP]. (2016). Cultura política de la democracia en las Américas, 2014: Gobernabilidad democrática a través de 10 años del Barómetro de las Américas.

Rey, T. E. (2006). A la vuelta de la esquina. La izquierda revolucionaria uruguaya 1955-1973. Montevideo, Uruguay: Fin de Siglo.

Rodríguez, A. (2017). Reflexiones sobre el concepto cultura política y la investigación histórica de la democracia en América Latina. Historia Y MEMORIA, (14), 205-247. doi:10.19053/20275137. n14.2017.5820

Sabetti, F. (2009). Democracy and Civic Culture. En Carles Boix y Susan Stokes (Eds.), The Oxford Handbook of Comparative Politics. Oxford, United Kingdom: Oxford University Press.

Santana, P. (1998). Opinión Pública, culturas políticas y democracia. Nómadas, (9), 83-93.

The Economist. (2016). Democracy Index 2016 Revenge of the "deplorables". The Economist Intelligence Unit. Recuperado de https://www.eiu.com/topic/democracy-index

Zapirain, H. (s.f.). Uruguay. Avances y retos del gobierno del Frente Amplio: Consejos de salarios y negociación colectiva. Recuperado de http://white.lim.ilo.org

\section{Notas}

${ }^{1}$ El grupo Contadora fue conformado en 1983 por México, Colombia, Venezuela y Panamá con el objetivo de buscar una salida negociada de los conflictos armados centroamericanos. De este proceso se derivan los Acuerdos de Esquipulas, cuyo fin era diseñar estrategias y establecer los cursos de acción para alcanzar la paz en la región
(Alba et al., 2016, p. 253). Algunas de las estrategias para mantener las negociaciones y diálogo constante entre las partes involucradas, se dieron con la instauración de una agenda que integraba obligaciones como: el retiro de asesores militares extranjeros en los países involucrados, el cese de venta de armamento y congelación del ya existente, la reactivación económica y la solución de las problemáticas regionales por medio de elementos democráticos que facilitan el consenso (Durán, 1984).

${ }^{2}$ La Constitución de Guatemala establece que la potestad del poder legislativo se concentra en el Congreso de la República, el cual se compone de un total de 158 diputados distribuidos así: "Cada uno de los departamentos de la república, constituye un distrito electoral. El municipio de Guatemala forma el distrito central, y los otros municipios del departamento de Guatemala constituyen el distrito de Guatemala. Por cada distrito electoral deberá elegirse como mínimo un diputado. La ley establece el número de diputados que correspondan a cada distrito en proporción a la población. Un número equivalente al veinticinco por ciento de diputados distritales será electo directamente como diputados por lista nacional" (Const., 1985, art. 157).

${ }^{3}$ En el Artículo 84 de la Constitución de Uruguay se establece la estructura de la Asamblea General, donde se estipula que este organismo estará compuesto por dos Cámaras: una de Representantes y otra de Senadores (Const., 1967, art. 84). La Cámara de Representantes se compondrá de noventa y nueve miembros elegidos directamente por el pueblo (Const., 1967, art. 88). Por su parte, la Cámara de Senadores se compondrá de treinta miembros, elegidos directamente por el pueblo, en una sola circunscripción electoral (Const., 1967, art. 94). 
